\title{
Stop-reaction time and the internal clock
}

\author{
LUC ROUSSEAU and ROBERT ROUSSEAU \\ Université Laval, Quebec, Quebec, Canada
}

\begin{abstract}
In a stop-reaction-time (stop-RT) task, a subject is presented with a regular, isochronous sequence of brief signals separated by a constant time interval, or stimulus onset asynchrony (SOA). His/her task is to press a response key as fast as possible when the sequence stops. As the sequence unfolds, an internal representation of the SOA duration builds up. Stop-RT is assumed to be triggered when an internal clock, operating as an "alarm clock," reaches a time criterion. Criterion setting is contingent upon variability in the SOA's internal representation. In Experiment 1A, stop-RT was measured for isochronous sequences of brief tones, light flashes, and also sequences of tones and flashes presented in regular alternation (tone-light-tone ...). Stop-RT was a linear function of SOA duration (ranging from 250 to $1,000 \mathrm{msec}$ ), regardless of modality, supporting a "central-clock" hypothesis. On the other hand, taken together, the results of Experiments $1 \mathrm{~A}, 1 \mathrm{~B}, 2$, and 3 suggest that no internal representation of the bimodal (tone-light) SOA of alternating sequences builds up. Indeed, an alternating sequence is physically equivalent to two interlaced isochronous subsequences, one auditory and one visual. So, two internal representations, one for the auditory (tone-tone) and one for the visual (light-light) SOA, could build up, and two time criteria running "in parallel" could thus support stopRT. To provide a critical test of parallel timing, stop-RT was measured for bimodal 5:3 polyrhythms formed by the superposition of auditory and visual isochronous sequences that had different SOA durations (Experiment 4). Parallel timing accounted for a large proportion of variance in polyrhythmic stop-RT data. Overall findings can be accounted for by assuming a functional architecture of an internal clock in which pulses emitted by a central pacemaker are available in parallel with two modalityspecific switch-accumulator "timing modules."
\end{abstract}

Most psychophysical models of time perception assume, implicitly or explicitly, the existence of an internal timekeeper, or "clock," common to both vision and audition (Allan, 1992). Data supporting that claim come from studies showing intermodality transfer of performance or correlation (Eijkman \& Vendrik, 1965; Loeb, Behar, \& Warm, 1966; Warm, Stutz, \& Vassolo, 1975). Others have interpreted differences in performance levels between modalities as an indication that timing was supported by modalityspecific timers, thus rejecting the idea of a central amodal timekeeper (Fraisse, 1952; Kolers \& Brewster, 1985).

However, differences in performance levels between modalities need not lead to such an interpretation. In the context of interval discrimination, Grondin (1993) reported large differences in $\Delta T_{75}$ for empty auditory and visual time intervals over a wide range of base durations $(.125$ to $4 \mathrm{sec})$. Despite such a difference in performance, when $\Delta T_{75}$ was

This research was supported by Grant A7921 from the Natural Sciences and Engineering Research Council of Canada awarded to R. R. It is based on a doctoral dissertation submitted by $L$. R. to Université Laval in partial fulfillment of the requirements for the $\mathrm{PhD}$ degree. We thank Arthur F. Kramer, Charles E. Collyer, and two anonymous reviewers for their helpful comments on the manuscript. Experiment 3 evolved from a conversation with Simon Grondin. We thank Chantal Belley for running subjects in Experiment 3. A preliminary report of Experiment 4 was made at the 25th International Congress of Psychology, July 19-24, 1992, Brussels. L. Rousseau is now at Centre de recherche, Centre hospitalier Côte-des-Neiges, Montreal. Correspondence concerning this article should be addressed to R. Rousseau, École de psychologie, Université Laval, Québec, Canada GIK 7P4 (e-mail: robert@psy.ulaval.ca). plotted against base duration, $T$ (a Weber function), similar slopes were obtained for auditory and visual functions, with a difference in intercept. That is to say, their data followed a generalized form of Weber's law (see Getty, 1975). Weber functions can be seen as a simple way to partition the contribution of two classes of processes to the total variance of a discrimination performance: one whose variance is a function of the base duration (time-dependent or scalar timing processes), and the other whose variance is time independent. Time-dependent processes selectively affect the slope of the Weber function, while time-independent processes affect the intercept (Church, 1978; Getty, 1975; Ivry \& Hazeltine, 1995). Following those lines, Grondin (1993) argued that the similarity of slopes for auditory and visual functions was an indication of a common timekeeping mechanism, while the difference in intercept was an indication of the contribution of nontemporal processes. Hocherman and Ben-Dov (1979), Grondin and Rousseau (1991), and Grondin (1993) have proposed that auditory and visual intervals have access to time-independent modality-specific encoding processes in addition to a central timing process. The lower intercept for auditory intervals was interpreted as an indication that auditory encoding of duration was more efficient than visual encoding. Thus, durationdiscrimination performance would be under the mixed control of a central timer and modality-specific encoding.

To bypass modality-specific processes and to gain "direct" access to a central, amodal internal clock, it has been suggested that bimodal empty time intervals, marked, for instance, by a short tone at onset and a light flash at offset, 
be used (Collyer, 1974; Rousseau \& Kristofferson, 1973). Admittedly, bimodal intervals accessing a central clock more "directly" would lead to optimal performance. Surprisingly, in duration-discrimination tasks, performance levels obtained under bimodal conditions have been shown to be much lower than those obtained with empty unimodal, auditory, or visual time intervals (Fraisse, 1952; Grondin \& Rousseau, 1991; Hocherman \& Ben-Dov, 1979; Rousseau, Poirier, \& Lemyre, 1983). At first view, such findings can be interpreted as detrimental to the notion of a central clock concept. Indeed, as suggested by Rousseau and Kristofferson (1973), unimodal intervals could provide access to more efficient modality-specific timing mechanisms. Moreover, Fraisse (1952) proposed, from similar results, that central timing does not obtain for short time intervals. The decrement observed in bimodal duration discrimination would reflect the transfer of the visual marker to the auditory modality, in order for duration to be timed within a modality-specific timer.

However, Rousseau et al. (1983) argued that this was not the case. They showed that the standard deviation of unimodal and bimodal psychometric functions was a linear function of the duration of the reference interval. That is to say, their data followed a generalized Weber's law. The slopes of the functions were quite similar for all marker conditions, while a much higher intercept was observed for bimodal intervals. Rousseau et al. argued that the similarity of slopes for bimodal and unimodal functions was an indication of a common timekeeping mechanism operating under all interval conditions, while the differences in intercept were an indication of the contribution of nontemporal processes. Most authors have considered the lower performance level with bimodal intervals as support for mixed timing interpretations (Grondin, 1993; Grondin \& Rousseau, 1991; Hocherman \& Ben-Dov, 1979; Rousseau \& Kristofferson, 1973). That is to say, both uni- and bimodal intervals are handled by a central timing mechanism, yet bimodal intervals are not supported by efficient modality-specific encoding processes, which would reduce the variability of the discrimination performance for unimodal intervals.

Rather than relying on access to efficient modalityspecific processes, Rousseau et al. (1983) argued that marking an interval with signals from different modalities induced more variability in the on/off triggering of a central timekeeper. Church (1978) had already proposed that the intercept of a Weber function could serve as a measure of the time required "to start and stop" an internal clock; both unimodal and bimodal duration discrimination would be under the control of a central clock, but bimodal intervals would induce a less efficient central on/off process, as suggested by the higher intercept value. For Grondin and Rousseau (1991), however, it was the lower efficiency of the decision-making processes involved in the short/long categorization of intervals which affected bimodal duration discrimination, with the nontemporal processes increasing variability of the discrimination performance for bimodal intervals. Finally, Hocherman and Ben-Dov (1979) proposed that unimodal intervals were supported by more efficient memory storage.
So bimodal intervals do not seem to provide simple access to a central clock in duration discrimination. A major difficulty lies in the interpretation of the nontemporal variance. We would like to propose that the duration-discrimination task in itself could be the limiting factor, and that bimodal intervals could give access to the central clock if used in another experimental task in which the relative contribution of task-specific nontemporal variability was reduced. The general aim of the present paper was thus to gain further support for a central clock by using bimodal intervals, and we chose to examine bimodal interval timing within a stop-reaction-time task.

In a typical stop-reaction-time (stop-RT) task (see ten Hoopen, 1985, for a review), a subject is presented with a regular, isochronous sequence of brief signals separated, from onset to onset, by a constant time interval, or stimulus onset asynchrony (SOA). The subject's task is to press a response key as fast as possible when the sequence stops. The number of signals varies from one sequence to another. Stop-RT is measured from the last signal's offset to keystroke. When compared with discrimination tasks, stopRT appears to be a simpler task. There is not much of a between-trial memory requirement. Furthermore, there is no need to classify intervals in order to select the appropriate response. Since the performance index in stop-RT is response latency, it does not readily relate to duration discrimination where response proportion is the performance index. However, it can be shown that both tasks can be supported by the same psychophysical internal-clock model. The model thus provides a bridge between these two different tasks.

Psychophysical timing models commonly assume that an internal representation of physical duration is obtained through the accumulation, over the extent of a time interval, of pulses emitted by a central pacemaker, or "clock" (e.g., Creelman, 1962; Divenyi \& Danner, 1977; Gibbon \& Church, 1984; Gibbon, Church, \& Meck, 1984; Killeen \& Fetterman, 1988; Killeen \& Weiss, 1987; Rousseau, Picard, \& Pitre, 1984; Treisman, 1963). These models, often collectively called internal-clock models (see Allan, 1992), assume the internal representation of a duration interval to be a random variable that follows some distribution (often normal). The mean of the distribution, $t$, is close to the actual duration, $T$, of the interval, and its total variance, $\operatorname{var}[t]$, is a combination (often additive) of the variance of different components of a given model. Similarly, stop-RT models (Schaefer, 1990; ten Hoopen \& Akerboom, 1983) propose that, during a sequence, an internal representation of the SOA duration builds up. This internal representation is assumed to be a normally distributed random variable (Schaefer, 1990).

Applying internal-clock models to stop-RT, it can be assumed that, following the first one, each signal in a sequence stops the pulse-accumulation process and, after storage of the accumulated value, starts accumulation for the next interval. The accumulated number of pulses is assumed to be a random variable, normally distributed with the mean, $t$, close to the actual duration, $T$, of the SOA, and variance, $\operatorname{var}[t]$. When accumulation reaches a $t$ value large 
enough so that the sequence can be considered as terminated (a time criterion), a stop-RT response is triggered. So stop-RT is not contingent on the occurrence of an external signal; it depends on the internal clock's operating as an "alarm clock." This is essentially equivalent to Kristofferson's (1977) real-time criterion model for duration discrimination, where a long response is triggered when the criterion is reached and a short response is triggered when the signal marking the offset of an interval occurs first. By comparison, stop-RT is simpler. The only event to trigger a response is when accumulation reaches a criterion value. No response is associated with the occurrence of a signal (each signal is simply assumed to reset the accumulation process). Reaching the criterion value is unambiguous information for response generation.

Optimal stop-RT performance is largely dependent on the appropriate setting of the criterion value on which the response depends. If it is set too close to the mean, a large number of responses will be triggered when a sequence has not actually ended (false alarms). Therefore, it has to be set at a value large enough to reduce false alarms to an acceptable level while enabling a fast response. Since the internal representation of the SOA duration is assumed to be a normally distributed random variable, the criterion is defined as a statistical criterion (Schaefer, 1990). It is assumed that a criterion, $C$, is set at a certain number, $n$, of standard deviations from the mean, $t$, of the internal distribution. The actual number depends on the accuracy level required by the task. So, the criterion can be defined as $C=" t+n(\operatorname{var}[t])^{1 / 2}$. Parameter $t$ will increase linearly as a function of SOA duration, $T$, with slope around 1 . As var $[t]$ increases, $C$ will increase and stop-RT will be longer. Experimental variables known to affect $\operatorname{var}[t]$ in duration discrimination are assumed to induce an increase in stop-RT.

As in duration discrimination, $\operatorname{var}[t]$ in stop-RT can be partitioned into two classes of sources of variance. Variables affecting scalar timing processes will affect the slope of the stop-RT function. Variance of nontemporal processes, which is independent of the SOA duration, will produce a change in the intercept of the function. Akerboom, ten Hoopen, Olierook, and van der Schaaf (1983) reported empirical evidence that gives a good indication that stopRT supports such a psychophysical modeling. They showed auditory stop-RT to increase linearly with SOA duration, with a slope of 1.12 for durations ranging from 40 to $2,130 \mathrm{msec}$. The fact that the slope was somewhat larger than 1 is consonant with Weber's law and is typical of the effect of time-dependent variables on psychophysical timing. Moreover, Akerboom et al. (1983) reported a constant increase in stop-RT $(25 \mathrm{msec})$ when tone signals were presented alternatively to one ear and then the other (interaurally) rather than to the same ear (monaurally). Interaural and monaural stop-RT functions had the same slope, 1.12, but their intercepts differed by $25 \mathrm{msec}$. This difference was independent of the SOA duration in the 40to 2,130-msec range, suggesting that presentation mode was a time-independent variable. These results parallel those reported for duration discrimination by Rousseau et al.
(1983). Indeed, they showed standard deviations (SDs) of the psychometric functions to vary linearly with interval duration, with a slope around .07 , in line with Weber's law. Similarly, presentation mode (bimodal vs. unimodal) of the interval affected $S D$. Bimodal intervals showed an $S D$ $70 \mathrm{msec}$ longer than that of unimodal intervals, a value that was constant over interval durations ranging from 250 to $1,000 \mathrm{msec}$.

In addition to SOA duration and presentation mode, stop-RT is known to be affected by the number of signals in a sequence. Stop-RT slowly decreases as a function of sequence length. In general, sequence length does not interact with presentation mode, except for very short sequences of two or three signals (ten Hoopen \& Akerboom, 1983). Moreover, Akerboom et al. (1983) found no systematic relationship between sequence length and presentation mode over a number of experiments.

In the present paper, we report a series of experiments in which stop-RT was measured for sequences of brief tones or light flashes (unimodal SOA) and sequences of tones and flashes presented in regular alternation (bimodal SOA). We tested several hypotheses about psychophysical timing models as applied to the stop-RT task. First, we sought to determine whether stop-RT would show the linear increase in RT with SOA that is predicted by timing models. In the course of that investigation, we discovered an interesting "sawtooth" pattern when bimodal stop-RT was plotted as a function of sequence length. Experiments $1 B$ and 2 were conducted to isolate experimental factors responsible for that effect. The data suggested that the pattern might result from the application of parallel unimodal timers to different components of a bimodal sequence, and Experiments 3 and 4 were conducted to test predictions derived from that hypothesis.

\section{EXPERIMENT 1A}

In this experiment, stop-RT data were obtained under a range of SOA durations comparable to that used by Rousseau et al. (1983) for duration discrimination (250$1,000 \mathrm{msec}$ ). It was intended as a test of the assumption that stop-RT could provide more direct access to the central clock than could duration discrimination. In its strongest form, the central-clock hypothesis predicts no difference between the three presentation modes (auditory, visual, bimodal). Actually, all three stop-RT functions should overlap with same slope and same intercept. On the other hand, if experimental variables known to affect variance in duration discrimination have a comparable effect on stopRT, stop-RT functions should replicate discrimination data. More specifically, unimodal and bimodal functions should be parallel and linear, with a slope somewhat larger than 1. Differences in intercept should also appear, with the bimodal function intercept being much larger than either unimodal function intercept. Parallel linear functions would provide a strong indication that timing was under the control of a central clock mechanism. However, if stopRT functions were to display important differences in slope, that would severely limit a central timing hypothesis. 


\section{Method}

\section{Subjects}

The subjects were eight 21 - to 28 -year-old ( $M=23.5$ ) volunteers ( 5 women and $3 \mathrm{men}$ ) who were paid $\$ 5$ per session. They were undergraduate and graduate students at Université Laval. All had normal or corrected-to-normal vision, and none reported any hearing defect. They were all naive as to the purpose of the experiment.

\section{Stimuli and Apparatus}

Auditory stimuli were $800-\mathrm{Hz}$ pure sine waves delivered through Telephonics TDH- 49 headphones at $80 \mathrm{~dB}$ (A) SPL. Each tone pulse lasted $10 \mathrm{msec}$ and had 2 -msec rise/fall amplitude ramps to avoid clicks. Auditory stimuli were generated by Coulbourn Instruments modules under software control. Visual stimuli were fast rise/fall light flashes with a luminance of $3 \mathrm{~cd} / \mathrm{m}^{2}$, delivered by a small neon bulb (Ne-40) inserted in a box located roughly $1 \mathrm{~m}$ from a subject's eyes $\left(.78^{\circ}\right.$ of arc). Each light flash lasted for $10 \mathrm{msec}$. The experiment was under computer control. A timing board (Metrabyte Dash-8) accessed with machine-language subroutines allowed response latencies to be recorded to the nearest millisecond.

\section{Design and Procedure}

Isochronous sequences, that is, regular sequences of brief signals separated, from onset to onset, by a constant time interval, or stimulus onset asynchrony (SOA), were used. The SOA duration was 250 , 625 , or $1,000 \mathrm{msec}$. The number of signals in a given sequence was $10,11,12,13,14,15$, or $n$. The $n$ value was randomly chosen from two numbers: 16 or 17 . Sequences of $n$ signals served as "dummy" trials to control for anticipatory responses based on a counting strategy for sequences of 15 signals, particularly at long SOA durations (Akerboom et al., 1983). The presentation mode of sequences varied according to the sensory modality of signals. In auditory and visual unimodal sequences, all signals were brief tones or light flashes. In bimodal sequences, auditory and visual signals were presented in regular alternation, beginning with a tone (tone-light-tone ...).

The subjects were tested individually in a sound-attenuated booth dimly lighted by an indirect lamp. The subject sat comfortably on a two-arm chair at a viewing distance of approximately $1 \mathrm{~m}$ from the light source. Written instructions in French were read to each subject. The following is a free translation:

In this experiment, you will be presented with sequences of brief tones, sequences of light flashes, and also sequences of alternating tones and flashes. In a given trial, one sequence is presented. Your task is to press the response button as quickly as possible when the sequence stops. If you ever respond before the end of a sequence, a FALSE ALARM sign will appear on the screen. Please try to react as fast as you can while avoiding false alarms.

The subject initiated a trial by depressing an 80-g inertia pushbutton with his/her dominant index finger, which triggered, after a constant 500-msec delay, the onset of the first signal of the sequence. The subject depressed the same pushbutton to indicate his/her response. Response latency (stop-RT) was computed from the offset of the last signal to the onset of the pushbutton relay. A computer monitor facing the subject displayed the word READY at the beginning of each trial; this was replaced by the word OK when a response was made after the last signal or by the words FALSE ALARM when the response was made before the last signal. No response-latency feedback was given. A response initiated a 2 -sec intertrial interval.

The subjects were familiarized with the stop-RT task during a short practice session. Each subject completed eight experimental sessions which lasted about $40 \mathrm{~min}$ each. Each session contained nine blocks ( 3 presentation modes $\times 3$ SOA durations) of 21 trials each ( 7 sequence lengths $\times 3$ replications). There were thus a total of 24 observations for each condition per subject. Blocks were separated by $10-\mathrm{sec}$ rests that the subject could prolong at will. Order of trials, blocks, and sessions was counterbalanced within and between subjects.

\section{Results}

Data from "dummy" trials were removed from the analysis. Stop-RTs under $100 \mathrm{msec}(0.54 \%$ of data $)$ or over $3 \mathrm{sec}(0.06 \%$ of data) were also removed from the analysis. Individual data were pooled over sessions. Mean stop-RTs averaged over subjects $(n=8)$ are shown in Figure 1 as a function of presentation mode and SOA duration.

A linear regression computed for each stop-RT function yielded slopes of $1.032,1.032$, and 1.050 for auditory, visual, and bimodal conditions, with intercepts of 225,270 , and $286 \mathrm{msec}$, respectively (all $r^{2} \mathrm{~s}$ exceeded 0.999 ). Mean stop-RTs were submitted to a 3 (presentation mode) $\times 3$ $(\mathrm{SOA}) \times 6$ (sequence length) three-way analysis of variance (ANOVA). This analysis yielded a significant main effect of presentation mode $\left[F(2,14)=64.47, M S_{\mathrm{e}}=\right.$ $2,963.21, p<.0001]$. The main effect of SOA was highly reliable $\left[F(2,14)=3,000.78, M S_{\mathrm{e}}=7,274.15, p<.0001\right]$. More critically, presentation mode did not interact with SOA $[F(4,28)<1]$, a finding that suggests parallel slopes for auditory, visual, and bimodal stop-RT functions (see Figure 1).

The main effect of sequence length was significant $\left[F(5,35)=47.09, M S_{\mathrm{e}}=1,268.40, p<.0001\right]$ (see Figure 2). The effect of sequence length on stop-RT was not consistent across SOA durations, as revealed by a reliable two-way interaction effect $\left[F(10,70)=4.63, M S_{\mathrm{e}}=772.24\right.$, $p<.0001]$. More importantly, while auditory and visual stop-RT exhibited a well-documented, slowly decreasing function (see Akerboom et al., 1983; ten Hoopen \& Akerboom, 1983), bimodal stop-RT followed a sawtooth-shaped function! Indeed, the effect of presentation mode on stopRT was qualified by a significant interaction with sequence length $\left[F(10,70)=9.27, M S_{\mathrm{e}}=1,153.68, p<.0001\right]$. The three-way interaction did not reach statistical significance $[F(20,140)<1]$.

\section{Discussion}

The slope of a stop-RT function is determined by scalar timing processes. Parallel slopes are assumed to support a

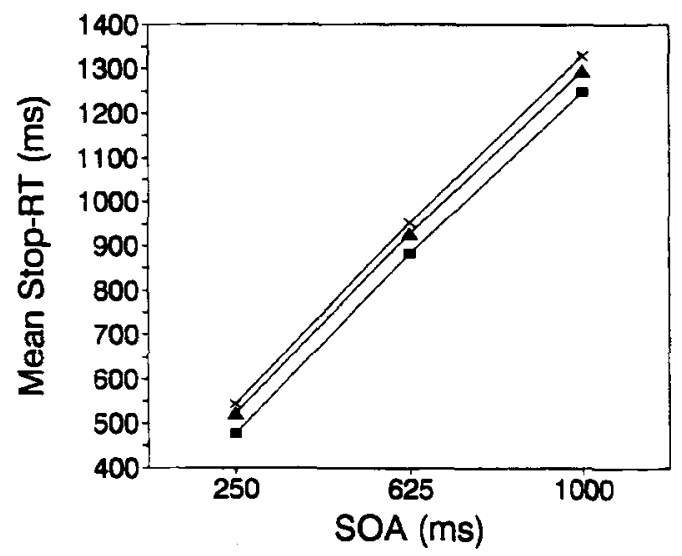

Figure 1. Mean stop-reaction time (in milliseconds) as a function of stimulus onset asynchrony for auditory (squares), visual (triangles), and bimodal $(\times$ s) sequences in Experiment $1 \mathrm{~A}$. Each data point represents about 1,152 observations. 

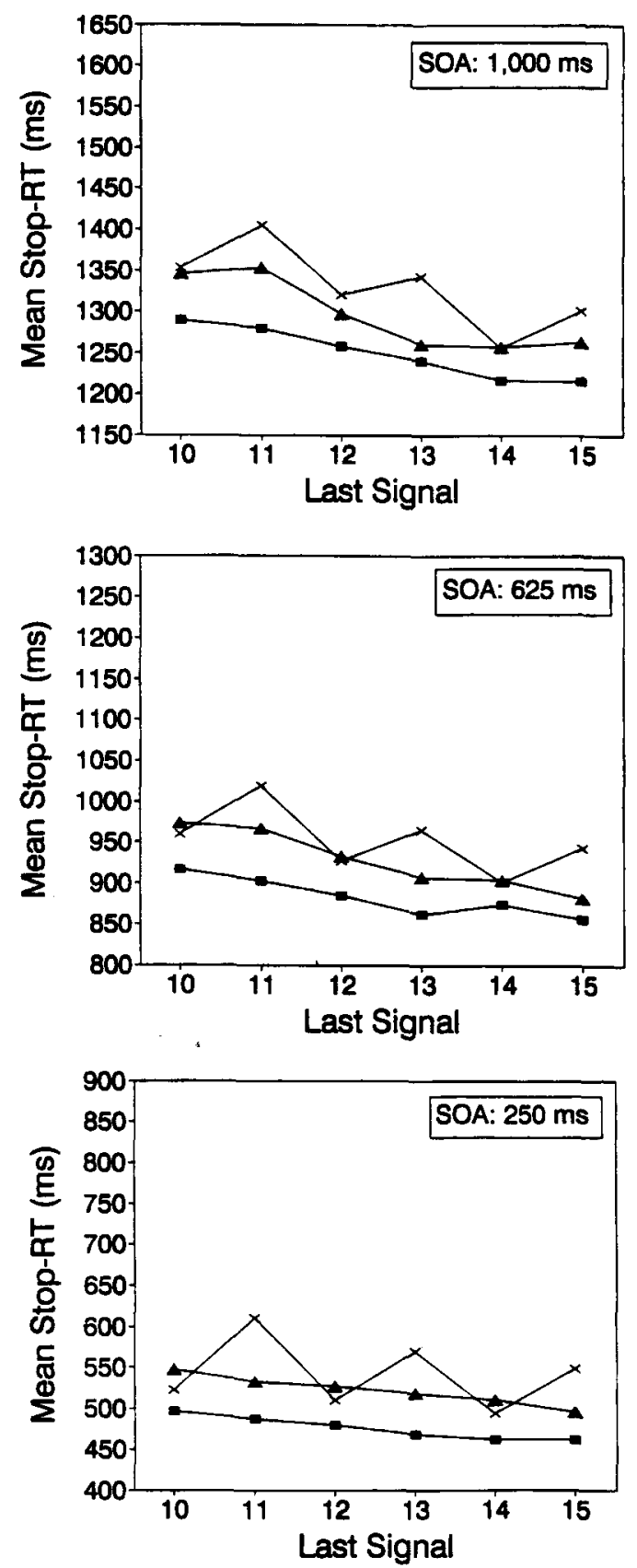

Figure 2. Mean stop-reaction time (in milliseconds) as a function of stimulus onset asynchrony and sequence length for auditory (squares), visual (triangles), and bimodal $(\times s)$ sequences in $\mathbf{E x}-$ periment $1 \mathrm{~A}$. Each data point represents about 192 observations.

central clock hypothesis. In this experiment, an identical slope (1.032) was found for auditory and visual stop-RT functions. Although the slope of the bimodal function (1.050) was slightly steeper, given the absence of an interaction between presentation mode and SOA duration, both uni- and bimodal conditions are assumed to have equivalent slopes. This finding suggests that a central timekeeper underlies stop-RT, regardless of presentation mode. However, these results do not support a strong case for central timing. Stop-RT functions did not overlap. The intercept of the bimodal stop-RT function was $16 \mathrm{msec}$ higher than the visual one, and the visual function was $45 \mathrm{msec}$ higher than the auditory one. Even though the ordering of the functions is the same as that in Rousseau et al.'s (1983) duration-discrimination data, the actual sizes of the differences in intercept are very different. The auditory/visual difference is larger than the unimodal/bimodal difference. Moreover, the main effect of presentation mode is tricky to interpret in terms of intercept, given a significant interaction with sequence length. A look at Figure 2 suggests that the auditory/visual stop-RT difference is independent of SOA duration and also constant over the range of sequence length. However, this is not the case for the unimodal/bimodal stopRT differences. Bimodal stop-RT is systematically faster when the number of presented signals is even $(10,12$, or $14)$ rather than odd $(11,13$, or 15$)$. In a bimodal sequence beginning with a tone, the last signal is a light for an even number of signals and conversely, a tone for an odd number of signals. Both even/odd and tone/light effects were highly unexpected. Although ten Hoopen and Akerboom (1983, Experiment 1) had previously observed a difference in auditory stop-RT between odd and even end signals, there is little empirical or theoretical support for an interpretation of these puzzling data. Therefore, to disentangle the relationship between presentation mode and sequence length, a control experiment was carried out in which number of signals and modality of the last signal were manipulated independently.

\section{EXPERIMENT 1B}

The purpose of this experiment was to highlight the critical factor underlying the sawtooth-shaped bimodal stop-RT versus sequence-length function observed in Experiment $1 \mathrm{~A}$. In addition to unimodal auditory and visual sequences, we measured stop-RT for bimodal sequences beginning with a tone, yielding a light at even numbers of signals, as in Experiment $1 \mathrm{~A}$, and also for bimodal sequences beginning with a light, yielding a tone at even numbers. If number (even/odd) of signals is the critical factor, then bimodal stop-RT should be faster for even than for odd numbers, regardless of the modality of the last signal.

\section{Method}

The method was identical to that of Experiment 1A, except as noted below.

\section{Subjects}

The subjects were 10 new, 19- to 34-year-old $(M=22.0)$ volunteers ( 7 women and 3 men) who were paid $\$ 5$ per session.

\section{Design and Procedure}

To the auditory, visual, and tone-light (tone as first signal) bimodal sequences, we added a fourth presentation mode; this was a light-tone (light as first signal) bimodal sequence. The SOA duration was $250 \mathrm{msec}$. The $n$ value was chosen randomly from four numbers: 8,9 , 16 , or 17 . The subject triggered a sequence by depressing a pushbutton and keeping it depressed. To indicate his/her response, the subject released the pushbutton. This new response mode was used 
to make sure that the subject always kept his/her finger in close contact with the pushbutton.

Each subject completed four experimental sessions, each of which lasted about $20 \mathrm{~min}$. Each session contained four blocks (four presentation modes) of 42 trials each ( 7 sequence lengths $\times 6$ replications). There were thus a total of 24 observations for each condition per subject.

\section{Results}

Data from "dummy" trials were removed from the analysis. Stop-RTs under $100 \mathrm{msec}(0.12 \%$ of data) or over $3 \mathrm{sec}$ (none) and false-alarm data $(2.14 \%$ of remaining data) were also removed from the analysis. Individual data were pooled over sessions. Mean stop-RTs averaged over subjects $(n=10)$ are shown in the left panel of Figure 3 as a function of presentation mode and sequence length.

As clearly shown in Figure 3, auditory stop-RT was faster than visual stop-RT, regardless of sequence length, a replication of the finding in Experiment $1 \mathrm{~A}$. The average difference between the two unimodal conditions was $67 \mathrm{msec}$. More critically, bimodal stop-RT was $84 \mathrm{msec}$ faster, on average, when the last signal was a light (even end signal 10,12 , or 14 of the tone-light sequence or odd end signal 11,13 , or 15 of the light-tone sequence) rather than a tone, regardless of the number of signals in a sequence. These results provide convincing evidence that the modality of the last signal was the critical factor underlying the sawtooth-shaped bimodal stop-RT versus sequence-length function observed in Experiment 1A. The even/odd distinction was thus clearly irrelevant.

\section{Discussion}

Stop-RT models assume that the RT is determined by timing the time elapsed since the occurrence of a signal in a sequence. Assuming that differences in stop-RT are related to changes in $\operatorname{var}[t]$, these results can be interpreted as indicating that, in bimodal sequences, timing an inter- val that begins with a light is less variable than timing one that begins with a tone. However, if we consider unimodal intervals, the opposite occurs. Intervals beginning with a light have a longer stop-RT than those beginning with a tone, an indication that timing is more variable for intervals beginning with a light signal. On the other hand, if one considers the expected modality of the missing signal, a much more coherent picture emerges. When a tone is expected as the next signal, timing should be less variable. That statement would apply equally to unimodal and bimodal sequences.

In bimodal sequences, expectation follows from regular alternation. A tone signal is always expected to occur following a light signal, and the other way around. In unimodal sequences, it is not possible to separate the effect of end signal and the effect of expected signal, since there is no change in signal modality. The next experiment was run to test the relative importance of the modality of the expected signal in bimodal sequences.

\section{EXPERIMENT 2}

For a missing signal to play a role in response triggering, its modality has to be derived from the regular structure of the sequence. On the other hand, last-signal modality is unlikely to depend on that factor. Accordingly, two types of bimodal sequences were investigated. In one case, the regular condition, modality alternated regularly from one signal to the other, as in the previous experiments. In the other case, signal modality was determined in a pseudorandom way. The modality of a given signal was equally likely to be visual or auditory, with the restriction that in a given sequence tones appeared as often as light flashes. Furthermore, in the random condition, end-signal modality was balanced over trials in the same way as in the regular condition. When regular alternation is eliminated, expected modality of the next signal is highly uncertain.
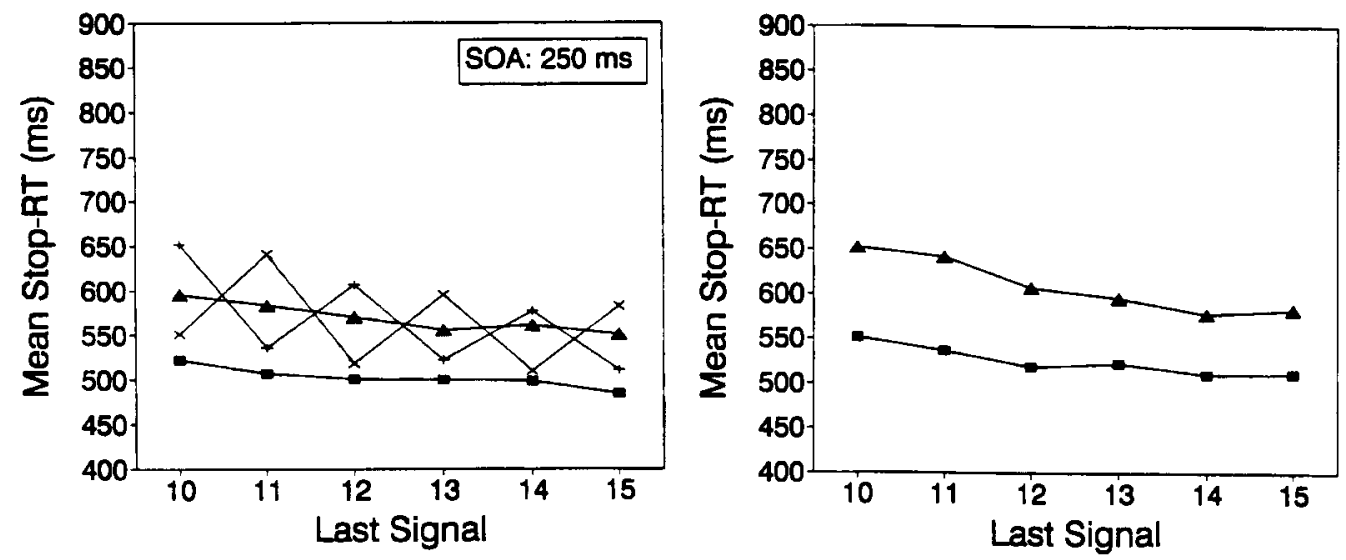

Figure 3. Left panel: Mean stop-reaction time (in milliseconds) as a function of sequence length for auditory (squares), visual (triangles), bimodal tone-light $(\times s)$ and bimodal light-tone $(+)$ sequences in Experiment 1 B. Each data point represents about 240 observations. Right panel: Bimodal stop-RT data from the left panel were replotted here as a function of the modality of the missing signal or, in other words, of the unimodal subsequence that stops for the auditory subsequence (squares) and the visual subsequence (triangles). 
So, if stop-RT is affected by the missing signal's expected modality, in the random-order condition, one should observe no effect of end-signal modality on stop-RT, that is, the sawtooth-shaped stop-RT function should disappear. Tone end-signal and light end-signal stop-RT functions should overlap.

\section{Method}

The method was identical to that of Experiment 1B, except as noted below.

\section{Subjects}

The subjects were 10 new, 21 - to 40 -year-old $(M=26.7)$ volunteers ( 5 men and 5 women), who were paid $\$ 5$ per session.

\section{Design and Procedure}

There were two presentation modes, both of which were bimodal. In regular-alternation sequences, tone and light signals were presented in regular alternation. In random-order sequences, an approximately equal number of tone and light signals were presented randomly. In a given block of the regular-alternation condition, $50 \%$ of the sequences began with a tone and $50 \%$ began with a light, so the last signal was a tone or a light with equal probability for each sequence length. In the random-order condition, the last signal was a tone or a light with equal probability for each sequence length.

Each subject completed four experimental sessions, each of which lasted about $20 \mathrm{~min}$. An experimental session contained four blocks ( 2 presentation modes $\times 2$ replications) of 42 trials each ( 7 sequence lengths $\times 2$ last signal modalities $\times 3$ replications). There were thus a total of 24 observations for each condition per subject.

\section{Results}

Data from dummy trials were removed from the analysis. Stop-RTs under $100 \mathrm{msec}(0.26 \%$ of data $)$ or over $3 \mathrm{sec}$ (none) and false-alarm data (3.36\% of remaining data) were also removed from the analysis. Individual data were pooled over blocks and sessions. Mean stop-RTs averaged over subjects $(n=10)$ are shown in Figure 4 as a function of presentation mode (regular alternation vs. random order), sequence length, and last signal modality. It is important to note that in Figure 4, stop-RT is presented as a function of last-signal modality without regard to firstsignal modality because in the random function there is no systematic relationship between first signal and last signal. It is as if all points corresponding to visual or auditory end signals in bimodal functions in Figure 3 were connected to each other. Thus, a sawtooth pattern should translate in Figure 4 as a constant difference between the visual and auditory functions.

Mean stop-RTs were submitted to a $2 \times 6 \times 2$ (presentation mode $\times$ sequence length $\times$ modality of last signal) three-way ANOVA. This analysis yielded a significant main effect of presentation mode $[F(1,9)=37.67$, $\left.M S_{\mathrm{e}}=7,583.62, p<.00025\right]$. Overall mean stop-RTs were 540 and $609 \mathrm{msec}$, respectively, for the regular-alternation and the random-order condition. The main effect of sequence length was reliable $\left[F(5,45)=61.42, M S_{\mathrm{e}}=\right.$ $510.73, p<.0001]$. Presentation mode interacted reliably with sequence length $\left[F(5,45)=10.12, M S_{\mathrm{e}}=247.00\right.$, $p<.0001]$. There was also a significant main effect of last signal modality $\left[F(1,9)=6.66, M S_{\mathrm{e}}=2,713.64, p<.03\right]$. More critically, presentation mode interacted significantly with modality of last signal $\left[F(1,9)=39.21, M S_{\mathrm{e}}=\right.$ $1,831.24, p<.0001]$. As can be seen in Figure 4, mean stopRT for the regular-alternation condition was reliably faster for light $(514 \mathrm{msec})$ than for tone $(566 \mathrm{msec})$ as last signal, a difference of $52 \mathrm{msec}$. For the random-order condition, the effect was the reverse; mean stop-RT was $600 \mathrm{msec}$ for tone as last signal and $617 \mathrm{msec}$ for light as last signal, but the difference was much smaller $(17 \mathrm{msec})$. The sequence length $\times$ last signal modality interaction was reliable $\left[F(5,45)=3.28, M S_{\mathrm{e}}=358.58, p<.015\right]$. The three-way interaction did not reach statistical significance $[F(5,45)=$ $2.21, p=.07]$.

Results clearly show that the critical factor is not the modality of the end signal as such. Randomizing tone and light signals not only reduced the effect of end-signal modality (from 52 to $17 \mathrm{msec}$ ); it even reversed it. Actually, the small effect of end-signal modality was now consonant with unimodal data.
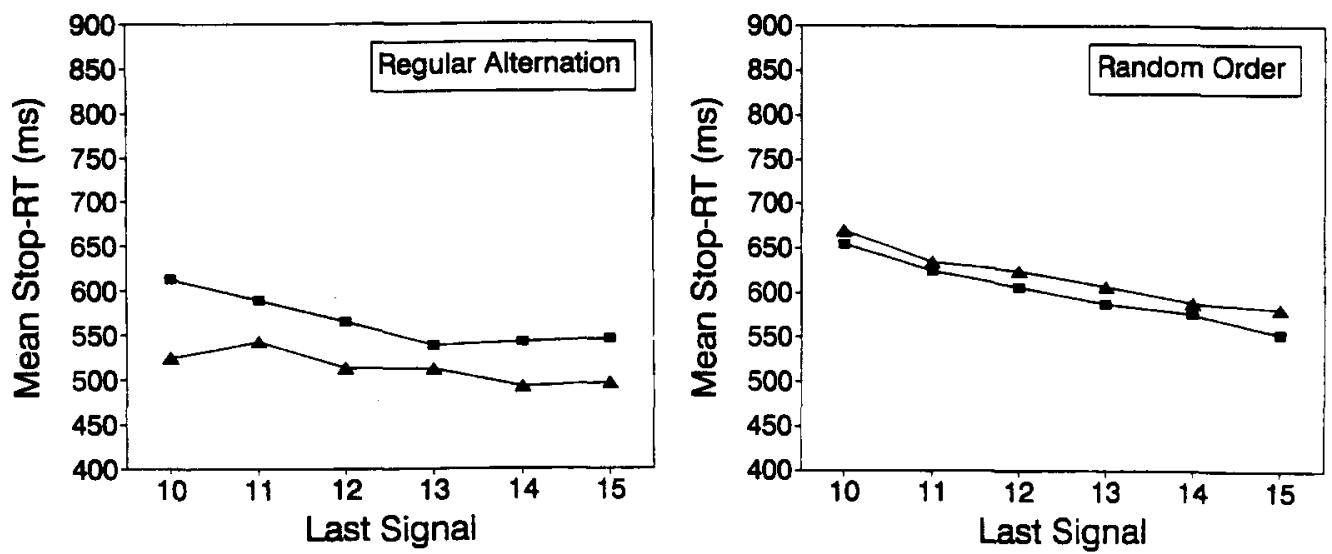

Figure 4. Mean stop-reaction time (in milliseconds) as a function of sequence length and modality of last signal (tone, squares; light, triangles) for regular-alternation and random-order bimodal sequences in Experiment 2. Each data point represents about 240 observations. 


\section{Discussion}

The importance of the expected modality of the missing signal strongly indicates that stop-RT is dependent on regularity of the sequence. Not only are the sequences isochronous, thus limiting temporal uncertainty, but signal modality alternation also reduces uncertainty concerning the stimulus structure of the sequence. It is difficult to accommodate the fact that modality of a missing signal has an effect on timing. How can timing be influenced by the modality of a missing signal? It could be possible that the regular alternation of modalities in bimodal sequences enables the development of two time criteria in the sequence, one for light-tone $(\mathrm{L}-\mathrm{T})$ intervals, beginning with a light flash and ending with a tone, and another for tone-light (T-L) intervals. These two criteria would operate alternately. The T-L intervals could induce a more variable internal representation of the SOA, causing a longer stopRT when a light is missing. However, this is not supported by bimodal duration-discrimination data. Rousseau et al. (1983) did not report any difference between discrimination of T-L and L-T intervals. Moreover, Grondin and Rousseau (1991) observed higher performance levels for T-L intervals, which would support a less variable internal representation.

We want to put forward that the influence of a missing signal in bimodal sequences could be better understood if these sequences were analyzed in a different way. Until this point, we considered stop-RT for bimodal sequences of regularly alternating auditory and visual signals to be based on the timing of bimodal ( $\mathrm{T}-\mathrm{L}$ or $\mathrm{L}-\mathrm{T}$ ) intervals. An alternative view is that it is based on the timing of two unimodal intervals, one auditory ( $\mathrm{T}-\mathrm{T}$ ) and the other visual $(\mathrm{L}-\mathrm{L})$. As shown in Figure 5, an alternating isochronous bimodal sequence is physically equivalent to two interlaced unimodal isochronous subsequences, one auditory and one visual. Each unimodal subsequence has a SOA duration twice that of the bimodal sequence, and one unimodal subsequence is shifted in time relative to the other by a duration corresponding to half the value of the bimodal SOA. Let us assume that stop-RT for bimodal sequences is handled by two modality-specific timers operating "in parallel" on the unimodal intervals rather than by an amodal central timekeeper operating on the bimodal intervals. In that case, a stop-RT response would be triggered simply when one or the other of the unimodal subsequences stopped. Stop-RT would show the properties of the unimodal timers associated with the expected modality of the missing signal. For timing purposes, the missing signal would not be related to the previous signal in the bimodal sequence (the last one) but would be to the previous signal in the unimodal subsequence of the same modality.

To illustrate the "parallel timing" hypothesis, bimodal data from Experiment 1B were replotted in the right panel of Figure 3 as a function of the modality of the missing signal or, in other words, of the unimodal subsequence that stops. As can easily be seen, two slowly decreasing stopRT functions, which nicely mimic those observed for unimodal conditions, are obtained, with bimodal stop-RT now shown to be faster when the auditory sequence stops. It follows that rather than a bimodal sequence's being timed by an amodal central internal clock as expected, it is controlled by two unimodal modality-specific timers. This is a major concern inasmuch as it would make the comparison of bimodal stop-RT with unimodal stop-RT meaningless. Actually, that comparison would provide information on the effect on stop-RT of having to time two unimodal sequences simultaneously. A strong version of parallel timing would assume no difference between stop-RT in the unimodal and bimodal contexts.

Actually, an examination of the right panel of Figure 3 suggests that this might not be the case, stop-RT always being longer in bimodal than in unimodal sequences for the same expected modality of the missing signal. However, one has to take into consideration that the SOA of unimodal subsequences in the bimodal sequence is twice the unimodal SOA. So, for instance, when conditionalized on the expected missing-signal modality, performance at a bimodal SOA of $250 \mathrm{msec}$ should be compared to a unimodal SOA of $500 \mathrm{msec}$. As previously argued, SOA duration is a major determinant of $\operatorname{var}[t]$. Since unimodal subsequences in bimodal sequences operate at a longer SOA, they should indeed show a longer stop-RT. However, when compared with the proper SOA values, bimodal and unimodal stop-RT should be equivalent according to a parallel timing hypothesis. Our aim with the next experiment was to test that hypothesis.

\section{EXPERIMENT 3}

In this experiment, stop-RT in a bimodal sequence was compared with stop-RT in the unimodal sequence resulting from the removal of signals from one modality in the bimodal sequence. Thus, the unimodal auditory sequence was simply a $\mathrm{T}-\mathrm{L}$ bimodal sequence from which the light signals were removed (see Figure 5). Conversely, the visual unimodal sequence was simply a $\mathrm{L}-\mathrm{T}$ bimodal sequence from which the tones were removed. The unimodal sequences and the unimodal subsequences embedded in bimodal sequences unfold identically in time. The only difference between the two conditions is that, in the bimodal condition, a signal from the other modality is inserted at mid-SOA.

\section{Method}

The method was identical to that of Experiment 1B, except as noted below.

\section{Subjects}

The subjects were 10 new 19- to 32-year-old ( $M=24.0$ ) volunteers ( 6 women and 4 men), who were paid $\$ 5$ per session.

\section{Design and Procedure}

There were four experimental conditions: unimodal auditory, unimodal visual, bimodal tone-light, and bimodal light-tone. The SOA durations were $500 \mathrm{msec}$ for each unimodal sequence and $250 \mathrm{msec}$ for each bimodal sequence. The number of signals in the bimodal sequences was $10,11,12,13,14,15$, or $n$. The $n$ value was chosen randomly from four numbers: $8,9,16$, or 17 . In a T-L bimodal sequence, 

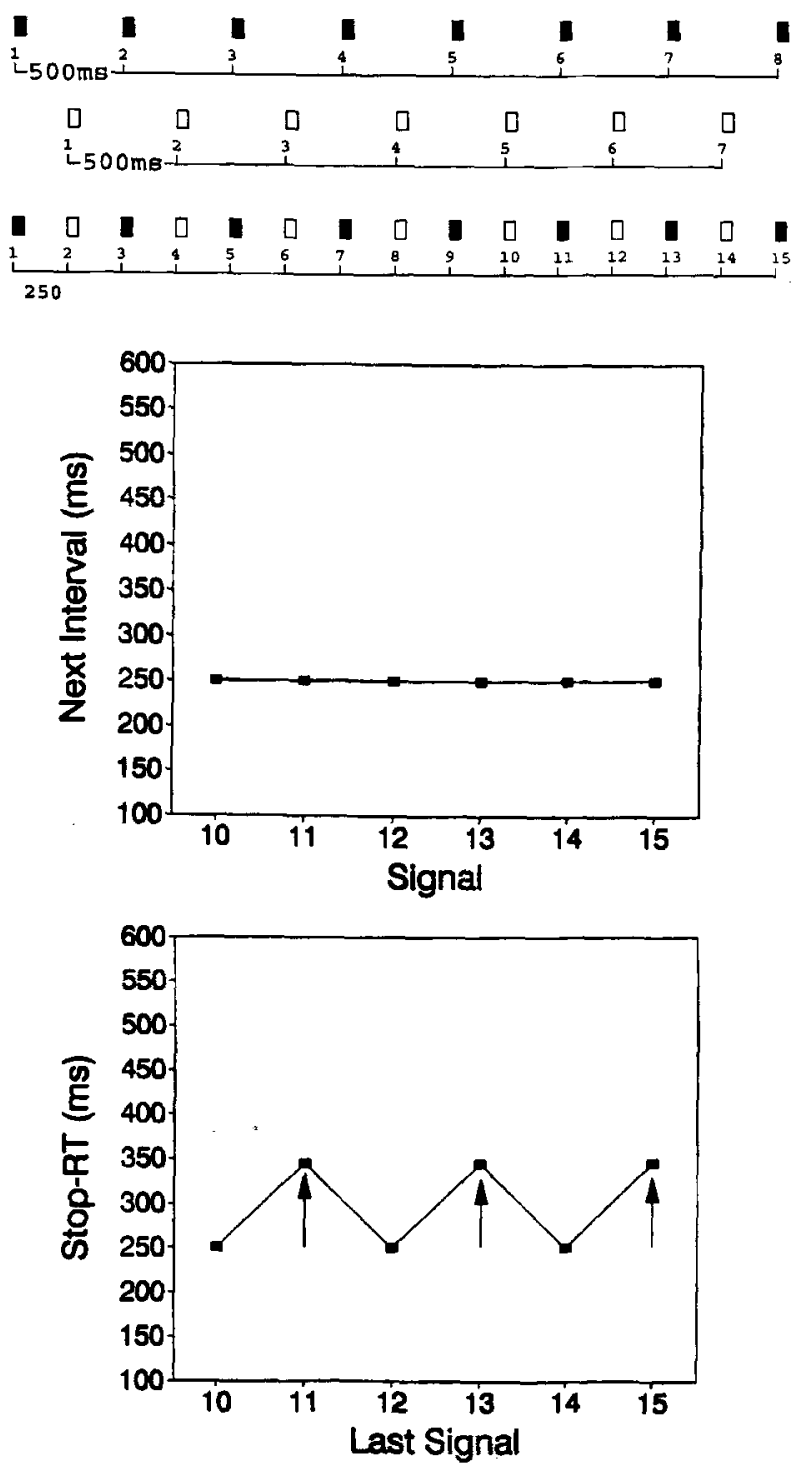

Figure 5. Top: Schematic of a bimodal sequence as the co-occurrence of two isochronous unimodal subsequences, one auditory (filled squares) and one visual (open squares). Middle: The physical temporal structure of the isochronous bimodal sequence. Each signal is followed by a 250 -msec SOA duration, yielding a flat pattern. Bottom: When the last signal of the bimodal sequence is a tone $(11,13$, or 15), so that the next light is missing, it is the visual sequence that stops. Given that unimodal visual stop-reaction time is slower than unimodal auditory stopRT (Experiments $1 A$ and 1B), increases in stop-RT (indicated by arrows) are expected to occur for last signals $(11,13$, and 15). This sawtooth-shaped bimodal stop-RT function is called a parallel timing "signature." Note that for a bimodal sequence beginning with a light (not illustrated), stop-RT increases correspond to End Signals 10, 12, and 14.

the auditory sequence stops when the last presented signal is a light (number 10,12 , or 14); similarly, in the L-T sequence, the visual sequence stops when the last presented signal is a tone (again, number 10,12 , or 14). It follows that unimodal subsequences stop after 5,6 , or 7 signals have been presented within that subsequence (see Figure 5). Accordingly, the number of signals in the unimodal sequence conditions was $5,6,7$, or $n$. The $n$ value was chosen randomly from two numbers: 8 or 9 . Unimodal sequence and subsequence stop-RTs are compared for unimodal number of signals ranging from 5 to 7 .

\section{Results}

Data from "dummy" trials were removed from the analysis. Stop-RTs under $100 \mathrm{msec}(0.12 \%$ of data) or over $3 \mathrm{sec}$ $(0.06 \%$ of data) and false-alarm data ( $2.07 \%$ of remaining data) were also removed from the analysis. Individual data were pooled over sequence lengths, blocks, and sessions. Mean stop-RTs averaged over subjects $(n=10)$ are shown in Figure 6 as a function of modality (auditory vs. visual) and presentation mode (unimodal vs. bimodal).

One-way ANOVAs were carried out separately for each modality. It revealed that auditory stop-RT did not differ between unimodal (769 msec) and bimodal (776 msec) presentation modes $[F(1,9)<1]$. By contrast, it indicated that visual stop-RT was significantly slower in the bimodal $(883 \mathrm{msec})$ than in the unimodal $(836 \mathrm{msec})$ presentation mode, an increase of $47 \mathrm{msec}\left[F(1,9)=11.34, M S_{\mathrm{e}}=\right.$ $978.79, p<.01]$.

\section{Discussion}

The present results give partial support to the paralleltiming hypothesis. While equivalence of unimodal and bimodal auditory stop-RT supports that hypothesis, that is not the case with the visual results. Bimodal visual stop$\mathrm{RT}$ is longer than unimodal visual stop-RT. There are a number of possible explanations for this discrepancy. However, before attempting to identify the source of the increase in visual stop-RT in bimodal sequences, it is necessary, given the mixed results of the present experiment, to consolidate the hypothesis that bimodal sequences are spontaneously split into unimodal subsequences.

A major limitation of the current test of the parallel timing hypothesis is the very high temporal coherence of the bimodal sequences. First, both unimodal subsequences have the same SOA. That could, for instance, enable the development of a single time criterion shared by both modalities. That criterion could preempt or be in addition to modalityspecific criteria. Moreover, the bimodal sequence has a SOA half the duration of the unimodal subsequence. That harmonic relation could, again, make possible the development of a bimodal criterion that could interact with other criteria. A number of authors have shown that the harmonic relationship between SOAs is critical in polyrhythmic tapping. In these tasks, subjects are asked to use one hand to tap in synchrony with an isochronous tone sequence and the other hand to synchronize with another isochronous sequence that has a different SOA. The ability of subjects to perform adequately in that parallel dual-tapping task is markedly reduced when the SOAs are not in a harmonic relationship (see, e.g., Summers, Rosenbaum, Burns, \& Ford, 1993). Thus, it is likely that temporal coherence of the regular modality alternation sequences does not make a strong test of the parallel timing hypothesis possible. The purpose of Experiment 4 was to provide a critical test of that hypothesis. 


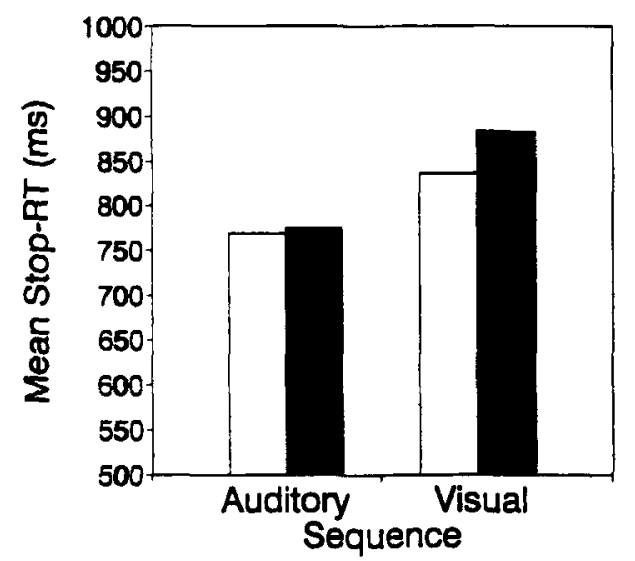

Figure 6. Mean stop-reaction time (in milliseconds) as a function of presentation mode (auditory vs. visual) of the isochronous sequence for unimodal (white bar) and bimodal (black bar) contexts in Experiment 3. Each bar represents about 720 observations.

\section{EXPERIMENT 4}

In this experiment, stop-RT was measured for bimodal sequences whose unimodal subsequences had different SOA durations. Different unimodal SOAs eliminate the possibility of a single time criterion's operating on both unimodal subsequences. If two time criteria could be shown to run simultaneously, a strong case would be made for parallel timing. To further break the temporal coherence of the bimodal sequence, the unimodal SOAs were chosen so as not to have a harmonic relationship. That is to say, one SOA was not an integer multiple of the other. One subsequence had a 750-msec SOA, and the other had a 450-msec SOA. Although each unimodal subsequence was rigorously isochronous, they formed a complex, anisochronous bimodal sequence - a 5:3 polyrhythm-when presented concurrently.

The bimodal $5: 3$ polyrhythm was formed by combining two unimodal isochronous subsequences, one containing five signals per pattern repetition and the other containing three signals per pattern repetition (see Figure 7). The two isochronous subsequences began simultaneously. In the $5(\mathrm{~T}): 3(\mathrm{~L})$ polyrhythmic pattern, there were five tone $(\mathrm{T})$ signals in the auditory subsequence and three light (L) signals in the visual subsequence. The $5(\mathrm{~L}): 3(\mathrm{~T})$ polyrhythm had the reverse pattern. The period of one pattern repetition cycle was $2,250 \mathrm{msec}$, so that the SOA duration of the fivesignal sequence was $450 \mathrm{msec}(2,250 / 5)$ and the SOA duration of the three-signal sequence was $750 \mathrm{msec}(2,250 / 3)$. One given repetition cycle contained seven successive signals, the first signal being a $T / L$ compound. Figure 7 illustrates the complex succession of SOA durations within the $5: 3$ polyrhythmic pattern. As can be seen, a bimodal sequence is, in fact, composed of three SOAs arranged in a complex manner. The SOA duration immediately following Signals 1,4 , and 7 is $450 \mathrm{msec}$, that following Signals 2 and 6 is $300 \mathrm{msec}$, and that following Signals 3 and 5 is $150 \mathrm{msec}$, forming the characteristic W-shaped tem- poral structure of a 5:3 polyrhythm (in comparison, an isochronous sequence has a flat pattern). A bimodal sequence of more than seven signals was formed by several repetition cycles.

Given different, nonharmonic unimodal SOAs and a complex bimodal SOA structure, if parallel timing obtains, timing in one unimodal subsequence will probably not interact with timing in the other subsequence or in the bimodal sequence. Stop-RT performance will be determined by known factors associated with unimodal timing in bimodal sequences. If we assume stop-RT to unimodal subsequences in polyrhythmic bimodal sequences to be controlled by parallel timing as in alternating bimodal sequences, it is possible to make predictions about performance from results of the previous experiments. Two main results of interest are that unimodal visual stop-RT is, on average, 45 msec longer than auditory stop-RT (Experiment 1 ) and that, while stop-RT to an auditory subsequence in a bimodal sequence is the same as that in a unimodal sequence, visual stop-RT is, on average, $50 \mathrm{msec}$ longer in a bimodal sequence (Experiment 3 ). Then, in a polyrhythmic bimodal sequence, if stop-RT to the auditory subsequence is taken as a base level, stop-RT to the visual subsequence is expected to increase by an amount equal to $95 \mathrm{msec}$, the total of unimodal (45-msec) and bimodal (50$\mathrm{msec}$ ) increases. Increasing stop-RT to the visual subsequence by $95 \mathrm{msec}$ produces systematic distortions in the standard W-shaped function, as seen in Figure 7. In the $5(\mathrm{~T}): 3(\mathrm{~L})$ polyrhythm, when the last signal is 2 or 5 , so that the next light (Signal 3 or Signal 6) is missing, it is the visual subsequence that stops. Consequently, stop-RT is predicted to increase by $95 \mathrm{msec}$ (indicated by arrows) at Last Signals 2 and 5. In the 5(L):3(T) opposite polyrhythm, when the last signal is $1,3,4$, or 6 , so that the next light (Signal $2,4,5$, or 7 ) is missing (the visual subsequence stops), the parallel-timing hypothesis predicts a $95-\mathrm{msec}$ increase in stop-RT (indicated by arrows) at Last Signals 1, 3, 4, and 6. Distorted W-shaped functions represented in Figure 7 are assumed to be parallel-timing "signatures," as sawtooth-shaped functions were assumed to be within the isochronous context of the previous experiments. These signatures provide explicit performance predictions of parallel timing within a polyrhythmic context.

\section{Method}

The method was identical to that of Experiment 1B, except as noted below.

\section{Subjects}

The subjects were 10 new, 20 - to 32 -year-old ( $M=25.4$ ) volunteers ( 6 men and 4 women), who were paid $\$ 5$ per session. Two subjects had had musical experience; 1 was an amateur pianist and the other had received piano lessons for a 5 -year period during his childhood.

\section{Design and Procedure}

The auditory and visual signals were identical to those used in the previous experiments. There were four polyrhythmic pattern types. In bimodal polyrhythms, the two isochronous subsequences were in opposite modalities: $5(\mathrm{~T}): 3(\mathrm{~L})$ or $5(\mathrm{~L}): 3(\mathrm{~T})$. In unimodal polyrhythms, 

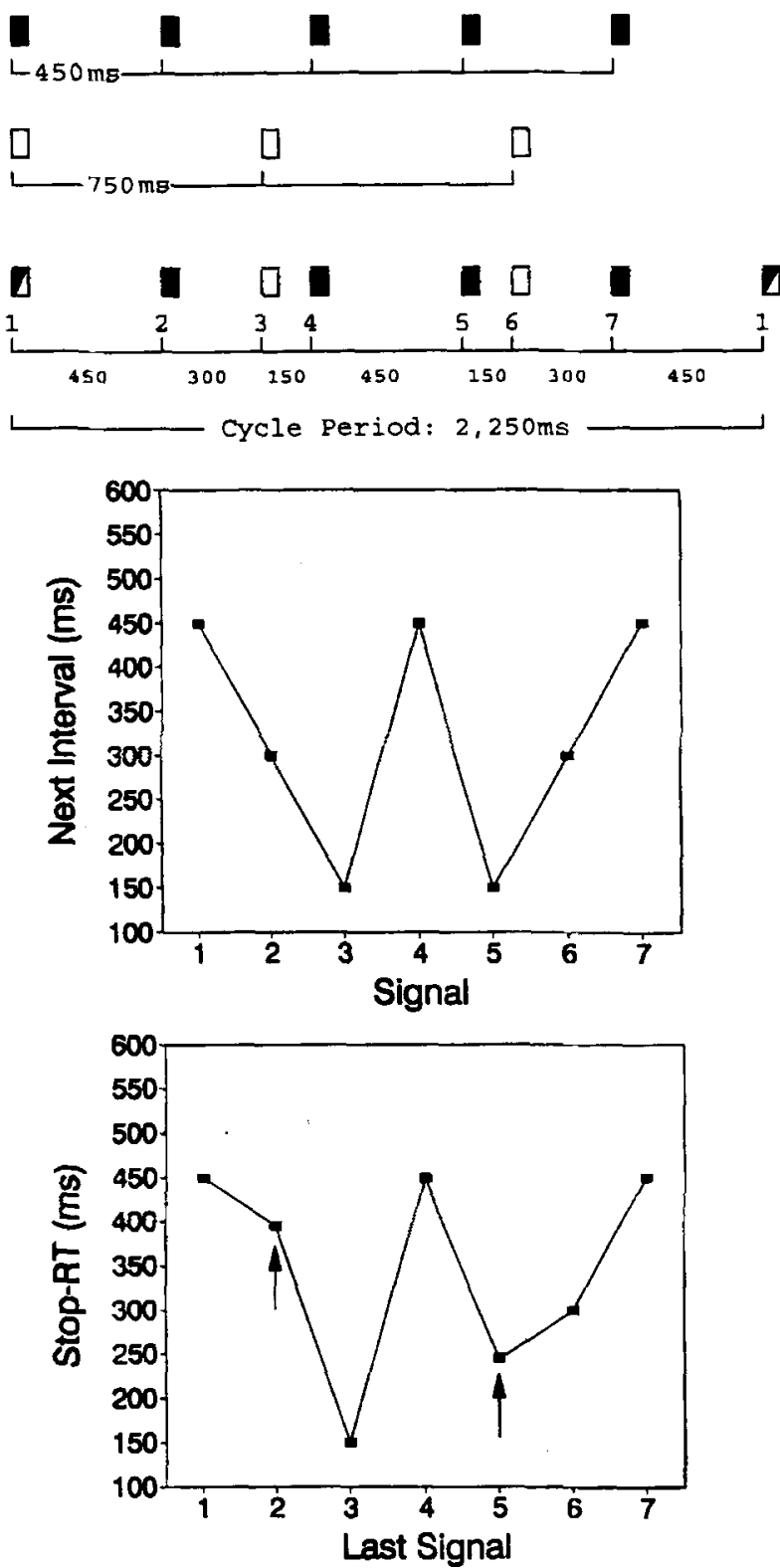
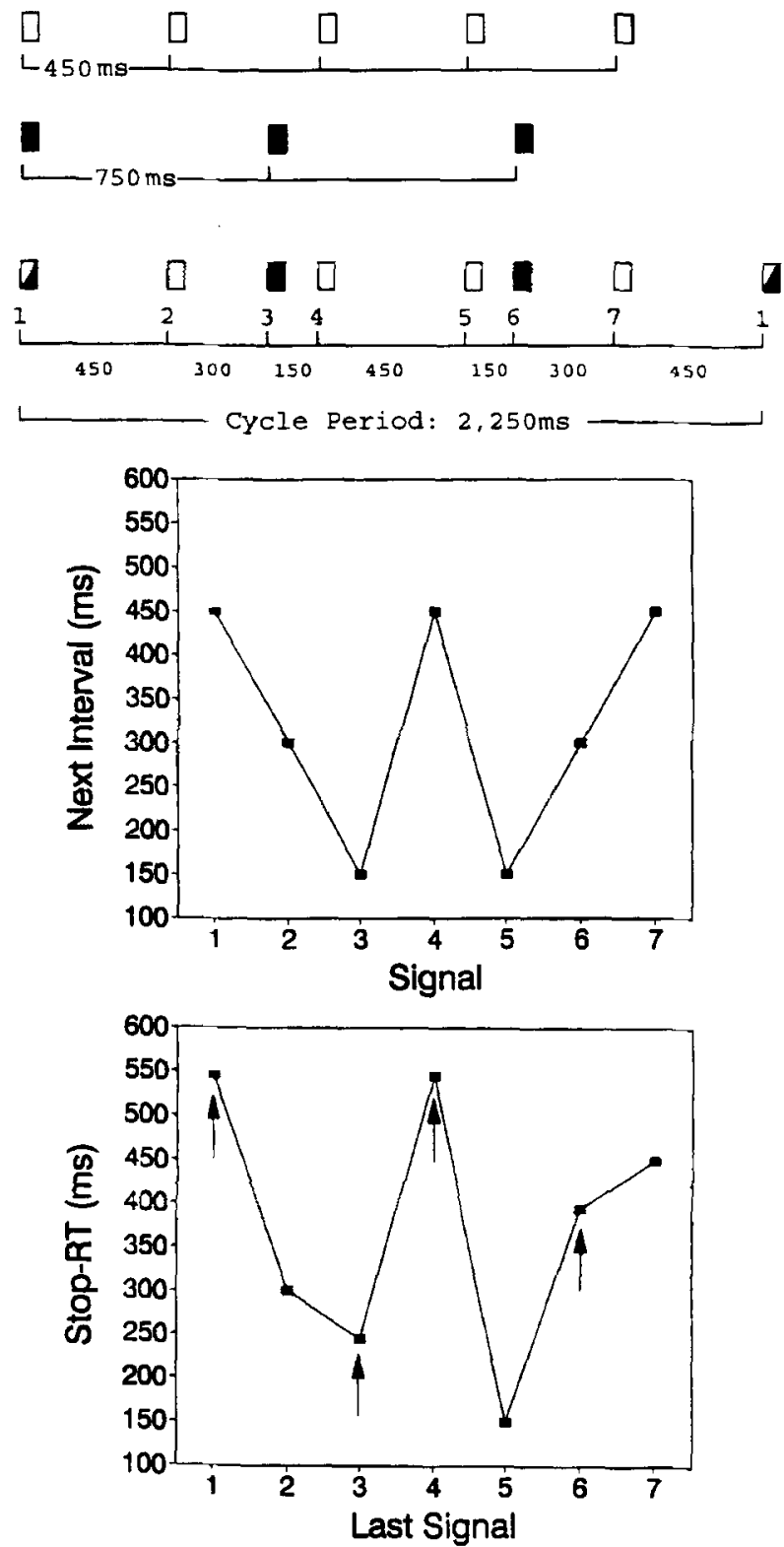

Figure 7. Top left: Schematic of a $5(\mathrm{~T}): 3(\mathrm{~L})$ bimodal polyrhythmic pattern, created by the superposition of two isochronous sequences, one with five tone signals (filled squares) per pattern repetition, and the other with three light signals (open squares) per pattern repetition. The SOA durations of the two sequences are 450 and $750 \mathrm{msec}$, respectively. One complete period $(2,250 \mathrm{msec})$ is shown, which includes the interval between the last signal of the pattern (the seventh one) and the first signal of the next period. Middle left: Schematic of the succession of SOA durations within one given repetition cycle of a 5:3 polyrhythm. The SOA duration immediately following Signals 1, 4, and 7 is $450 \mathrm{msec}$, that following Signals 2 and 6 is $300 \mathrm{msec}$, and that following Signals 3 and 5 is 150 msec, forming the characteristic W-shaped temporal structure of a 5:3 polyrhythm. Bottom left: Parallel timing "signature." Predicted increases in stop-RT are indicated by arrows (see text for explanations). Top right: Schematic of a 5(L):3(T) bimodal polyrhythmic pattern. Middle right: The characteristic W-shaped temporal structure of a 5:3 polyrhythm. Bottom right: Parallel-timing "signature."

even though all signals were identical, they are described in terms of two unimodal identical subsequences, auditory, $5(\mathrm{~T}): 3(\mathrm{~T})$, and visual, $3(\mathrm{~L}): 5(\mathrm{~L})$. Tones were delivered binaurally, at equal duration, frequency, and amplitude. Light flashes were emitted from a single, central source and were of equal luminance.

Practice session. Each subject was familiarized with the stop-RT task during a practice session that lasted about 10 min. A 3:2 polyrhythm was used. The period of one pattern-repetition cycle was $1,200 \mathrm{msec}$, so the SOA duration of the three-signal sequence was
$400 \mathrm{msec}(1,200 / 3)$ and the SOA duration of the two-signal sequence was $600 \mathrm{msec}(1,200 / 2)$. The following four pattern types were presented in consecutive blocks of trials: $3(\mathrm{~T}): 2(\mathrm{~T}), 3(\mathrm{~L}): 2(\mathrm{~L})$, $3(\mathrm{~T}): 2(\mathrm{~L})$, and $3(\mathrm{~L}): 2(\mathrm{~T})$. Each block contained 12 trials; the polyrhythm stopped once at each of the four possible last signals of the fourth, fifth, and sixth repetition cycle.

Experimental sessions. Each subject completed six experimental sessions which lasted about $30 \mathrm{~min}$ each. A given session contained four blocks (four pattern types) of 30 trials each. Of the 30 tri- 
als, 28 were experimental trials in which the polyrhythm stopped during the third or fourth repetition cycle ( 7 last signals $\times 2$ repetition cycles $\times 2$ replications) and 2 were "dummy" trials in which the polyrhythm stopped once at a randomly chosen end signal of the second repetition cycle and once at a randomly chosen end signal of the fifth repetition cycle. There were thus a total of 24 observations for each condition per subject.

\section{Results and Discussion}

Data from the "dummy" trials were removed from the analysis. Stop-RTs under $100 \mathrm{msec}(0.08 \%$ of data) or over $3 \mathrm{sec}(0.03 \%$ of data) and false-alarm data $(1.12 \%$ of remaining data) were also removed from the analysis. Individual data were pooled over repetition cycles and sessions. Mean stop-RTs averaged over subjects $(n=10)$ are shown in Figure 8 as a function of presentation mode and last signal number ( 1 to 7 ).

\section{Bimodal Polyrhythms}

As can be seen in Figure 8, observed polyrhythmic stopRT functions show typical W-shaped distortions predicted from parallel timing. In the $5(\mathrm{~T}): 3(\mathrm{~L})$ pattern, stop-RT is indeed increasing when the visual sequence stops, that is, when the last signal was 2 or 5 . In the 5(L):3(T) opposite pattern, stop-RT is also increasing when the visual sequence stops, that is, when the last signal was $1,3,4$, or 6 . Goodness of fit of the parallel timing predictions has been estimated from linear regression between parallel timing signatures and observed bimodal stop-RT functions (averaged over subjects). The coefficients of determination $\left(r^{2}\right)$, which index the proportion of variance in stop-RT data accounted for by parallel timing, were .83 and .89 for the $5(\mathrm{~T}): 3(\mathrm{~L})$ and $5(\mathrm{~L}): 3(\mathrm{~T})$ patterns, respectively. A closer inspection of Figure 8 shows that predictions from parallel timing are even better than might be indicated by the $r^{2}$ estimates. Actually, only one point in each function diverges markedly from the predictions: Point 5 in the 5(T):3(L) function and Point 3 in the $5(\mathrm{~L}): 3(\mathrm{~T})$ function. These are the only two points at which the visual sequence stops $150 \mathrm{msec}$ after a tone signal. Thus, here again, visual stop-RT seems to be particularly sensitive to the bimodal context. Overall, these data are taken as strong support for the parallel-timing hypothesis.
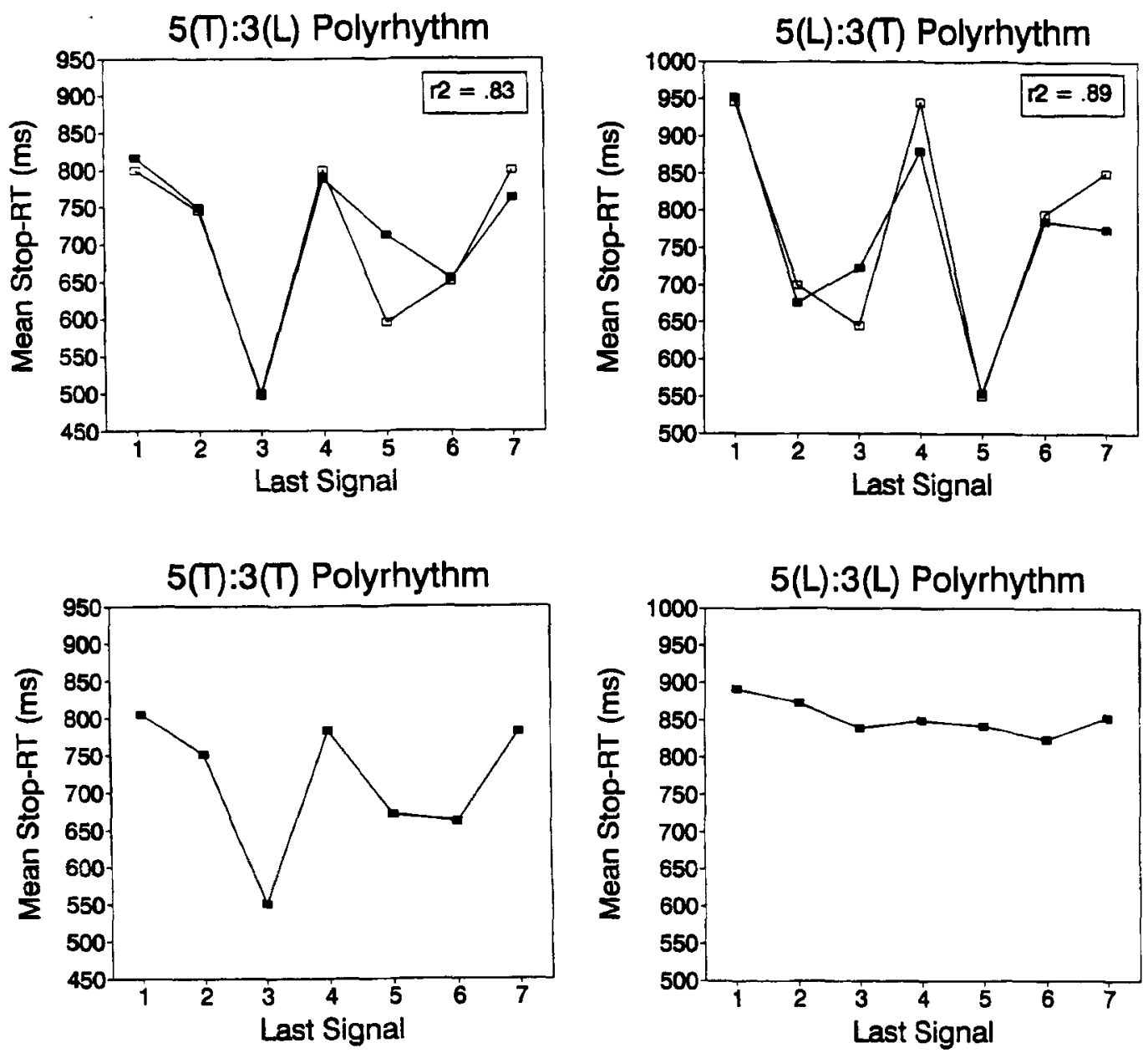

Figure 8. Observed stop-reaction time (in milliseconds) as a function of polyrhythmic pattern type and last signal number, in Experiment 4 (filled squares). Each data point represents about 240 observations. Parallel-timing "signatures" (open squares) are superimposed by eye upon observed bimodal stop-RT functions. 


\section{Unimodal Polyrhythms}

Surprisingly enough, as can be seen in Figure 8, the $5(\mathrm{~T}): 3(\mathrm{~T})$ auditory stop-RT function displayed typical parallel W-shaped distortions; the function is very similar to the 5(T):3(L) bimodal stop-RT function. When the last tone signal is 2 or 5 , so that the next tone (Signal 3 or Signal 6) of the "slow" (750 msec) isochronous subsequence is missing (the "slow" tone subsequence stops), stop-RT increases in a way that is similar to that found when a visual sequence stops within a bimodal polyrhythm. This similarity led us to suppose that the "fast" ( $450 \mathrm{msec})$ and "slow" $750 \mathrm{msec}$ ) isochronous tone subsequences could possibly be timed in parallel. The slow sequence timing is assumed to behave like visual sequence timing. Hence, stop-RT is assumed to increase by $50 \mathrm{msec}$ for a slow tone sequence presented concurrently with a fast tone sequence. Since, in this case, there is no increase in stop-RT due to auditory/visual differences, parallel-timing predictions can be obtained by adding $50 \mathrm{msec}$ when the last signal is 2 or 5 , that is, when the slow subsequence stops. The coefficient of determination $\left(r^{2}\right)$ between this paralleltiming prediction and the observed auditory polyrhythmic stop-RT function (averaged over subjects) was as high as .89. Such a finding, indicating that polyrhythmic auditory patterns might be timed as two concurrent auditory subsequences, is counterintuitive. Indeed, since the tones were physically identical in the auditory polyrhythm, no sensory cue was available to perceptually differentiate the two underlying isochronous sequences.

In contrast, the 5(L):3(L) visual polyrhythmic stop-RT function did not show any resemblance to either bimodal stop-RT functions or a straight $\mathrm{W}$ shape. This is at least as puzzling as the unimodal polyrhythmic auditory stop-RT results. Timing of visual polyrhythmic patterns is comparable to that of the unimodal isochronous sequences studied in the previous experiments. A flat visual stop-RT function was observed. The visual stop-RT function appears to be controlled by a single time criterion even though at least three SOA values are present in a polyrhythmic pattern. It is as if visual timing cannot support the development of more than one time criterion.

\section{GENERAL DISCUSSION}

The aim of the present paper was to gain further support for a general-purpose central timekeeping mechanism, or "internal clock," through bimodal stimulation within a stop-RT task. Assuming stop-RT to be under the control of psychophysical timing, it was expected that stop-RT would increase linearly with SOA duration and that nontemporal task-dependent variability would be reduced relative to that of duration-discrimination tasks.

Experiment 1A showed auditory, visual, and bimodal stop-RT to increase linearly with SOA duration (ranging from 250 to $1,000 \mathrm{msec}$ ) with equivalent slopes around 1.04 . Parallel slopes support psychophysical modeling of stopRT data and suggest that all interval types share a common, central pacemaker. Furthermore, Experiment 1A showed that unimodal visual stop-RT was 45 msec longer than uni- modal auditory stop-RT across all SOA values. This systematic difference in intercept can be interpreted as evidence for a more variable internal representation of a duration interval marked by visual rather than auditory signals. The fact that the auditory/visual difference occurred in the intercept of the stop-RT function and not in the time-dependent slope parameter suggests that sensory modality contributes a nontemporal source of variance to the internal duration representation. Grondin (1993) has reached the same conclusion in the context of duration discrimination. Interpreting bimodal stop-RT data turned out to be more complicated. On the one hand, bimodal stop-RT was comparable to visual stop-RT, both being longer than auditory stop-RT. This may suggest that stop-RT indeed reduced the nontemporal variability previously observed in duration discrimination. However, bimodal stop-RT turned out to be longer when the sequence ended with a tone than when it ended with a light flash. In Experiment 2 , it was shown that the critical variable was not the modality of the last signal in the sequence but, rather, the expected modality of the missing signal. We were then faced with a basic question: were bimodal sequences processed as a single sequence of bimodal intervals or as two concurrent unimodal subsequences? Support for concurrent processing accumulated over experiments. Experiment 3 provided evidence that timing an auditory interval in a bimodal sequence was identical to timing a similar interval in a unimodal sequence. However, visual timing deteriorated in a bimodal context. Visual stop-RT was slowed by $50 \mathrm{msec}$ as compared with unimodal sequence timing. Finally, in Experiment 4, stop-RT to polyrhythmic bimodal patterns was shown to conform to predictions based on the unimodal timing of visual and auditory isochronous subsequences. These data converge toward parallel timing of unimodal subsequences in bimodal sequences. Bimodal sequences would be split into two unimodal subsequences timed concurrently.

The concept of parallel timing is not without precedent in the literature on the psychophysics of time. Meck and Church (1984) reported evidence for the simultaneous timing of two signals, one auditory and one visual, by rats. Such findings had major theoretical implications for internalclock models. They led Church (1984) to state that for a single-pacemaker conception to be maintained, "there must be multiple switch-accumulator modules to handle simultaneous temporal processing" (p. 580). Olton, Wenk, Church, and Meck (1988) further proposed that attention could be divided between the outputs of the different switchaccumulator modules. A sequential attentional system would integrate decision processes in order to obtain response selection and production. Our conception of a functional architecture that supports parallel timing follows their theoretical position. A central pacemaker emits pulses that are available to modality-specific switch-accumulator timing modules. The common slope of stop-RT versus SOA functions for visual, auditory, and bimodal sequences supports the existence of a central, common pacemaker. When signals are presented in a bimodal isochronous sequence, successive tone signals are assumed to operate the switch 
of an auditory accumulator and successive light signals are assumed to operate the switch of a visual accumulator. Timing operations in the auditory and visual modules are assumed to take place simultaneously and independently of each other so that two internal time criteria, one auditory and one visual, can run in parallel without interference. When a given criterion is reached, the corresponding accumulator delivers an output signal for response generation. A serial monitoring system is assumed to control stop-RT response triggering. ${ }^{1}$ It monitors the occurrence of an output signal in a given module. The paralleltiming model bears some resemblance to the theoretical proposal put forward by Rousseau et al. (1983). They proposed that temporal information was central and common to all timing in a processing space, and that it was tapped when two events marking an interval occurred in the same "place," defined here as modules. The present model goes one step further by assuming that timing is able to operate concurrently in more than one module.

Data from Experiments 1A, 1B, 3, and 4 give a clear indication that although timing can be performed in parallel, there are basic differences in timing efficacy between modules. Visual timing is more variable than auditory timing. Furthermore, it is sensitive to the concurrent occurrence of an auditory sequence. Although the data do not provide a ready explanation for visual sensitivity to the bimodal context, a number of interpretations can be suggested. For instance, it could be that timing of the visual subsequence is disrupted by the regular occurrence of the tone during the visual interval, whereas auditory timing would be unaffected by light flashes. Interval timing has been shown to be affected by a signal presentation during the interval (Rousseau, Poirier, \& Tremblay, 1984; Vroon, 1973). The disruption would increase variability in visual timing, and would be most apparent in bimodal polyrhythms when a tone occurred $150 \mathrm{msec}$ before the missing light flash. It is also possible that there is a time-sharing cost associated with the concurrent timing of a visual unimodal sequence with an auditory sequence. Finally, the slowing down of visual stop-RT in bimodal sequences might come from visual timing's being less efficient at triggering a response.

Polyrhythmic unimodal visual and auditory stop-RT data in Experiment 4 provides insights into the capacity of the modules to handle more than one time criterion. The visual module behaves as if it were able to handle only a single criterion. However, if spatial cues were available to support the distinctiveness of two isochronous visual sequences, for instance by using two adjacent light sources, parallel timing might possibly be promoted. Indeed, according to Fraisse (1981), the basic difference between auditory and visual rhythmic timing performances is likely to diminish when the temporal structure of light signals is supported by a spatial structure. On the other hand, the auditory module appears to be able to handle two time criteria. Auditory polyrhythmic stop-RT data suggest that two isochronous tone sequences can be timed in parallel, even though there is no sensory basis (e.g., pitch) for their per- ceptual differentiation. It challenges a conception of timing modules as being modality specific. It is as if the timing system was able, without support other than temporal, to break a complex temporal pattern into more elementarythat is, isochronous - sequences. However, more research will be needed before such theoretical extensions can be considered. Undoubtedly, the open issue of multiple internal time criteria will eventually contribute to a better understanding of the functional architecture of an internal clock in terms of a central pacemaker subserving several switch-accumulator "timing modules."

\section{REFERENCES}

akerboom, S., ten Hoopen, G., Olierook, P., \& van der Schaaf, T. (1983). Auditory spatial alternation transforms auditory time. Journal of Experimental Psychology: Human Perception \& Performance, 9 , 882-897.

AllaN, L. G. (1992). The internal clock revisited. In F. Macar, V. Pouthas, \& W. J. Friedman (Eds.), Time, action and cognition: Towards bridging the gap (pp. 191-202). Dordrecht: Kluwer.

Church, R. M. (1978). The internal clock. In S. H. Hulse, H. Fowler, \& W. K. Honig (Eds.), Cognitive processes in animal behavior (pp. 277 310). Hillsdale, NJ: Erlbaum.

Church, R. M. (1984). Properties of the internal clock. In J. Gibbon \& L. Allan (Eds.), Timing and time perception (Annals of the New York Academy of Sciences: Vol. 423, pp. 566-582). New York: New York Academy of Sciences.

Collyer, C. E. (1974). The detection of a temporal gap between two disparate stimuli. Perception \& Psychophysics, 16, 96-100.

Creelman, C. D. (1962). Human discrimination of auditory duration. Journal of the Acoustical Society of America, 34, 582-593.

DivenYI, P. L., \& DANNER, W. F. (1977). Discrimination of time intervals marked by brief acoustic pulses of various intensities and spectra. Perception \& Psychophysics, 21, 125-142.

EIJkMan, E., \& VendriK, A. J. H. (1965). Can a sensory system be specified by its internal noise? Journal of the Acoustical Society of America, 37, 1102-1109.

Fraisse, P. (1952). La perception de la durée comme organisation du successif [Perception of duration as the organization of successiveness]. Année Psychologique, 52, 39-46.

Fraisse, P. (1981). Multisensory aspects of rhythm. In R. D. Walk \& H. L. Pick, Jr. (Eds.), Intersensory perception and sensory integration (pp. 217-248). New York: Plenum.

GeTTY, D. J. (1975). Discrimination of short temporal intervals: A comparison of two models. Perception \& Psychophysics, 18, 1-8.

GibBon, J., \& ChuRCh, R. M. (1984). Sources of variance in an information processing theory of timing. In H. L. Roitblat, T. G. Bever, \& H. S. Terrace (Eds.), Animal cognition (pp. 465-488). Hillsdale, NJ: Erlbaum.

Gibbon, J., Church, R. M., \& Meck, W. H. (1984). Scalar timing in memory. In J. Gibbon \& L. Allan (Eds.), Timing and time perception (Annals of the New York Academy of Sciences: Vol. 423, pp. 193197). New York: New York Academy of Sciences.

Grondin, S. (1993). Duration discrimination of empty and filled intervals marked by auditory and visual stimuli. Perception \& Psychophysics, 54, 383-394.

Grondin, S., \& RousseaU, R. (1991). Judging the relative duration of multimodal short empty time intervals. Perception \& Psychophysics, 49, 245-256.

Hocherman, S., \& Ben-Dov, G. (1979). Modality-specific effects on discrimination of short empty time intervals. Perceptual \& Motor Skills, 48, 807-814.

IVRY, R. B., \& Hazeltine, R. E. (1995). Perception and production of temporal intervals across a range of durations: Evidence for a common timing mechanism. Journal of Experimental Psychology: Human Perception \& Performance, 21, 3-18.

Killeen, P. R., \& Fetterman, G. (1988). A behavioral theory of timing. Psychological Review, 95, 274-295. 
KIlleEN, P. R., \& Weiss, N. A. (1987). Optimal timing and the Weber function. Psychological Review, 94, 455-468.

Kolers, P. A., \& Brewster, J. M. (1985). Rhythms and responses. Journal of Experimental Psychology: Human Perception \& Performance, 11, 150-167.

KRISTOFFERSON, A. B. (1977). A real-time criterion theory of duration discrimination. Perception \& Psychophysics, 21, 105-117.

Loeb, M., Behar, I., \& WARM, J. S. (1966). Cross-modal correlations of the perceived durations of auditory and visual stimuli. Psychonomic Science, 6, 87.

Meck, W. H., \& ChURCh, R. M. (1984). Simultaneous temporal processing. Journal of Experimental Psychology: Animal Behavior Processes, 10, 1-29.

Olton, D. S., Wenk, G. L., Church, R. M., \& Meck, W. H. (1988). Attention and the frontal cortex as examined by simultaneous temporal processing. Neuropsychologia, 26, 307-318.

Rousseau, R., \& Kristofferson, A. B. (1973). The discrimination of bimodal temporal gaps. Bulletin of the Psychonomic Society, 1, 115-116.

Rousseau, R., Picard, D., \& Pitre, E. (1984). An adaptive counter model for time estimation. In J. Gibbon \& L. Allan (Eds.), Timing and time perception (Annals of the New York Academy of Sciences: Vol. 423, pp. 639-642). New York: New York Academy of Sciences.

Rousseau, R., PoIrier, J., \& Lemyre, L. (1983). Duration discrimination of empty time intervals marked by intermodal pulses. Perception \& Psychophysics, 34, 541-548.

Rousseau, R., Polrier, J., \& Tremblay, G. (1984). Temporal organization and intermodality in duration discrimination of short empty intervals. In J. Gibbon \& L. Allan (Eds.), Timing and time perception (Annals of the New York Academy of Sciences: Vol. 423, pp. 636638). New York: New York Academy of Sciences.

SCHAEFER, F. (1990). The effect of system response times on temporal predictability of work flow in human-computer interaction. Human Performance, 3, 173-186.
Sorkin, R. D., BogGS, G. J., \& Brady, S. L. (1982). Discrimination of temporal jitter in patterned sequences of tones. Journal of Experimental Psychology: Human Perception \& Performance, 8, 46-57.

Summers, J. J., Rosenbaum, D. A., Burns, B. D., \& Ford, S. K. (1993). Production of polyrhythms. Journal of Experimental Psychology: Human Perception \& Performance, 19, 416-428.

TEN HOOPEN, G. (1985). The detection of anisochrony in monaural and interaural sequences. In J. A. Michon \& J. L. Jackson (Eds.), Time, mind, and behavior (pp. 140-150). Berlin: Springer-Verlag.

TEN HoOPEN, G., \& AKERBoOM, S. (1983). The subjective tempo difference between interaural and monaural sequences as a function of sequence length. Perception \& Psychophysics, 34, 465-469.

Treisman, M. (1963). Temporal discrimination and the indifference interval: Implications for a model of the "internal clock." Psychological Monographs: General \& Applied, 77(13, Whole No. 576).

VROON, P. A. (1973). Tapping rate as a measure of expectancy in terms of response and attention limitation. Journal of Experimental Psychology, 101, 183-185.

Warm, J. S., StUTz, R. M., \& VAssolo, P. A. (1975). Intermodal transfer in temporal discrimination. Perception \& Psychophysics, 18, 281-286.

\section{NOTE}

1. This view is conceptually related to the "separated channels" model presented in Figure 3 of Sorkin, Boggs, and Brady's (1982) paper on the discrimination of temporal jitter.

(Manuscript received March 7, 1994; revision accepted for publication August 21, 1995.) 